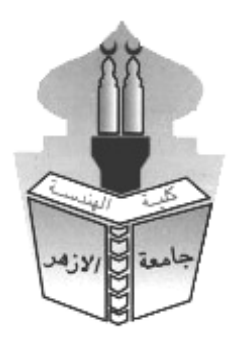

\title{
PERSUASION BASED RECOMMENDATION SYSTEM
}

\author{
Sarah Mahmoud, Reda Abo Alez and Fawzy EL-Refai \\ Department of System and Computer Engineering, El-AZhar University, Cairo, Egypt
}

\begin{abstract}
.
Persuasive role in Recommendation Systems operates like motivators influencing users to buy the recommended items. In this study, we rely upon the well established explanations of six Cialdini's Principles to enrich recommendation system with explanations and examine their effect on the persuasive power of recommendation item. The results of the experiment revealed that all six Cialdini's Principles positively affect user's influnce about the recommended book while reciprocity and authority seem to be the more effective ones. These indicate that a user's intention to purchase a recommended item is increased if the item is accompanied with a persuasive explanation.
\end{abstract}

\section{KEY WORDS: Persuasion, Recommender Systems, Cialdini’s Principles}

\section{INTRODUCTION}

Recommendation systems take user's preferences and interests in order to filter available information and then to provide them recommendation items that match their tastes [1].All the past researches in recommendation systems have traditionally been focused on their algorithmic aspect and more specifically evaluation of algorithms that provide accurate recommendations. The assumption that accuracy of the algorithm is the most significant factor that affects the quality and eventually the acceptance of a recommendation system has been recently challenged since other factors that play also a significant role have emerged. Explanations are an important factor of a recommendation system that may increase its acceptance by the user. The aim of this study is to examine if certain persuasive strategies (applied in the form of recommendation explanations) affect user's adoption of recommendations. The rest of the paper is organized as follows: Section (2) background definition related persuasive in recommendation system. section (3) recommendation system section (4) The Persuasive Role Of Explanations. The proposed algorithm is presented in section (5) along with the assumptions upon which the proposed technique is built. Section (6) shows performance evaluation of the proposed system using a realistic dataset. Finally, the whole paper is concluded in section (5).

\section{RELATED WORK}

Recently, there are many researches in recommendation systems. The mainstream of researches in Recommendation Systems have traditionally been focused on their algorithmic aspect and more specifically on the development and evaluation of algorithms that provide accurate recommendations items [2].In general, these researches can be those that do not use The persuasive role in recommendation systems or those that are based on the persuasive role in recommendation systems. In 1998, the author in [3] affect the persuasive ability of a recommendation defined as 'the attempt of changing people's attitudes or behaviours or both'. In 2007, the author in [4][5], discussed the influence of a number of explanation characteristics in the behavior of the system such as transparency, scrutability, trust, persuasiveness, effectiveness, efficiency and satisfaction. All of these characteristics can help to increase the system trustworthiness. In 2009 , the author in [6] proposed a prototype for a movie recommender 
system that is used to evaluate users' satisfaction in a number of explanation styles. In 2011, the author in [7] evaluated a number of explanation attributes such as satisfaction, efficiency, effectiveness, and trust in recommendation systems through comparing the performance of a number of explanation styles. Finally, in 2014, the author in [8], investigates if certain persuasive strategies in the form of recommendation explanations can affect user's adoption of recommendations items.

\section{RECOMMENDATION SYSTEMS}

The goal of any recommendation system is to generate relevant recommendations items for users. A Recommendation system is an information filtering process that helps users by filtering data and delivers relevant information to the users. An information system is known as a recommendation system engine when the delivered information comes in the form of suggestions. Since different users have different interests and preferences so the information filtering process must be personalized to accommodate the individual users interests. This requires gathering of users feedbacks in order to make a user profile of his interests and preferences. In the recent years, recommendation systems have become extremely common and they are applied to a variety of fields. Recommendation systems produce a list of recommendations items through collaborative or content-based filtering techniques. One of recommendation systems applications is to help users for finding which products they would like to purchase at E-Commerce sites. In general, recommendation systems follow a specific process to produce product recommendations. Nowadays, recommendation systems have achieved widespread success in the E- commerce field. An example of recommendation systems is Amazon.com which suggests books to users based on other books that users told Amazon they are interested in. Another example for recommendation systems is CDnow which helps users to choose which CDs to purchase. There are mainly three approaches for the information filtering in recommendation systems: Content-based filtering or collaborative filtering and hybrid filtering. Content-based filtering recommends items based on a comparison between the content of the items and a user profile. Collaborative filtering is used by some recommendation systems such as large commercial E-commerce sites. Collaborative filtering produces recommendations by computing the similarity between users preferences and the preferences of other people. Such recommendation systems do not analyze or understand the content of the items being recommended. Lastly ,hybrid filtering combines collaborative filtering and content-based filtering[1] .

\section{EXPLANATIONS IN RECOMMENDATION SYSTEMS}

One of the goals of explanations according to persuade users to try or purchase the item that is recommended to them. In general, persuasion can lead a user to change his attitudes or behaviours that lead to a better lifestyle [4]. For instance, if we want smokers to quit smoking they need to be persuaded. Explanations have an important role on Recommendation Systems since an explanation is a means through which a user perceives the value of the recommended item to decide whether it is close to his interests or not [9]. Explanations can operate like motivators and can be used by several systems such as Amazon and MovieLens [10]. However, there is no clear indication about what type of explanations can actually lead to persuasion, while still there is no enough empirical guide that demonstrates what type of influence strategy could lead to persuasion [11].

\section{THE PROPOSED SYSTEM}

In this section, the proposed system is introduced in detail. The application domain of our study is the book recommendation system. Firstly we design persuasive explanations, following Kaptein's methodology [11]. Six textual explanations were created in total, i.e. one for each Cialdini's Persuasion Principle. The content of each explanation was developed to comply with the main purpose of each Persuasion Principle. Finally, the six? (one for each strategy), were used in the experiment (Table 1). 
PERSUASION BASED RECOMMENDATION SYSTEM

Table 1. Explanation on each persuasion principle

\begin{tabular}{|c|c|}
\hline $\begin{array}{c}\text { Influence } \\
\text { Strategy }\end{array}$ & Explanations \\
\hline Reciprocity & A Facebook friend, who read the book that you suggested him/her in past recommends \\
you this book
\end{tabular}

For the purpose of the research, a book recommendation system was developed. At the first step of the research, users evaluated (through 1-5 ratings) a set of books, in order to have an adequate number of ratings for each user to produce recommendations based on the collaborative filtering algorithm.

At the second step, the Recommendation System provided a recommendations enriched with persuasive explanations. More specifically, a collaborative filtering algorithm was implemented and produced ratings for each of the items that the user has declared that he has not seen in the past. As mentioned above, the recommended book was enriched with persuasive explanations, based on Cialdini's Principles (the explanations from the first step of the research) and was reassessed from participants to examine which strategies influenced users to change their intention to read the recommended book or not. Each strategy was evaluated separately (through rating from1-5). The difference between the initial rating at the first step and the rating on each strategy denotes the persuasive effect of every strategy.

\section{EXPERIMENTAL RESULT AND PERFORMANCE EVALUATION}

In this section, we investigate the effectiveness of our proposed approach when applied on the real dataset.

\subsection{Study area and data description}

The proposed system described in section 5 is applied to a real dataset collected which collected from the Amazon product data which is an online site. This site contains dataset from Amazon which including 142.8 million reviews spanning May 1996 - July2014 [12]. The dataset contains books dataset reviews in the following format:

\section{UserID , BookID ,Rating}

- UserID Contains the ID of user who provided rating.

- BookID Contains the Book ID.

- Rating Contains the book rating information with a scale from 1-5 (rating 5 denoting higher rating and rating 1 denoting lower rating)

\subsection{Results and performance evaluation}

This section is devoted to evaluating the performance of the proposed system in section 5. Two performance metrics are considered. The first is to evaluate performance of a recommendation system in a given dataset. Mahout has implemented recommendation system such as User-User Collaborative Filtering and Item-Item Collaborative Filtering. For the purposes of this research, we will use User-User Collaborative Filtering [13]. The User-User CF creates a neighborhood of users that are similar to user based on similarity algorithms. Then by using neighborhood of users, the recommendation system estimates the user's preference for item by taking into consideration all of the preferences of neighbors in that have rated item. Finally User-User CF therefore focuses on similarities between users' preferences [12].The Mahout library implemented several similarity algorithms and allow to use them into the CF Recommendation system in order to identify similar neighborhoods for users or calculate similarities between items[16]. For the purposes of this research we will compare between the Pearson Correlation 
Coefficient Similarity and the Log Likelihood Ratio Similarity algorithms to get similarity algorithm with high accuracy. The Pearson Correlation Coefficient (PCC) determines the similarity between two users or items by measuring the tendency of two series of preferences to move together in a proportional and linear manner. The Log Likelihood (LL) relies on calculating similarity between two users or items based on statistics that revolve around occurrences related to these users or items [14][15]. LL focuses on events where users or items overlap in preferences, events where both users or items have preferences where and the compared user or item does not finally events where both users or items do not have preferences. We will use Root Mean Square Error (RMSE) to compare between two similarity. RMSE are known as predictive accuracy metrics because they represent how accurately a recommendation system estimates a user's preference for the item. In our book dataset RMSE will evaluate how the recommendation system can predict a user's rating for a book based on a scale rating from one to five stars. The smaller RMSE means the more accurate a recommendation system because RMSE will calculate smaller values if the deviations between actual and predicted ratings are smaller.The following results used. Table 2 and Chart 1 represent evaluation of User-User CF algorithms with both Pearson and Log Likelihood similarity algorithms.

Table 2 Comparison between Pearson Correlation Coefficient and Log Likelihood

\begin{tabular}{|l|l|l|}
\hline Metric & Pearson Correlation Coefficient & Log Likelihood \\
\hline RMSE & 5.4 & 3.5 \\
\hline Precision & 0.4 & 0.5 \\
\hline
\end{tabular}

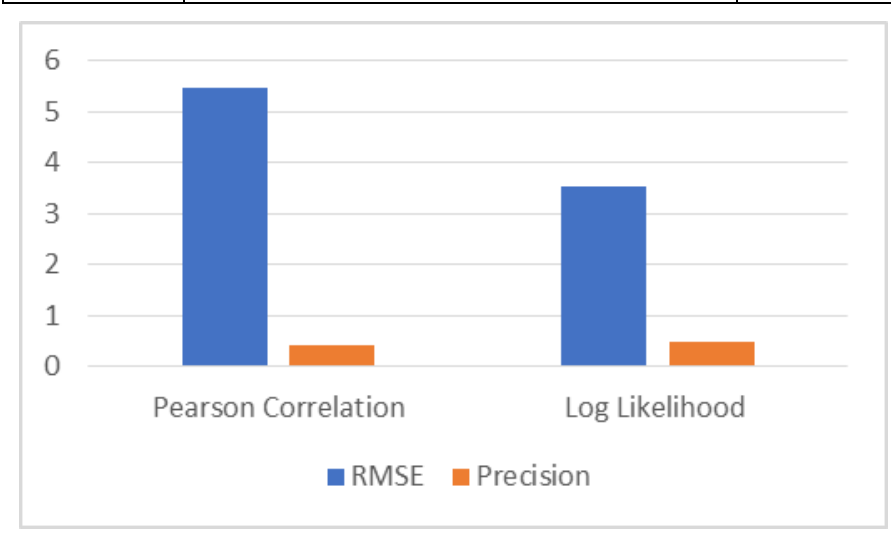

Fig. 1 Evaluation of User-User RMSE and Precision

Fig.2 shows that Log Likelihood has low RMSE and high precision So when User-User CF using the Log Likelihood similarity is the most accurate similarity algorithm .Another performance metric is to evaluate performance of using explanation role in recommendation system. The analysis of data was held using the statistical software SPSS [8]. First, we examined if users' behavior changed by comparing the averaged value of the initial rating that users provided for the book that was finally recommended to them with the average value of the ratings after the application of the influence strategies. The results demonstrate that on average there is a statistically significant change in the user's intention to read the book. To identify which strategies perform better in terms of persuasiveness, paired t-tests were used upon the differences between the initial rating and the one for each strategy. Paired t-test was used to examine significance, where 0.05 is set as the threshold for pvalue to evaluate the significance and $\mathrm{p}$-value lower than 0.001 indicates strong significance as shown in table 3. 
Table 3. Paired t-test results

\begin{tabular}{|l|c|}
\hline Strategy & p-value \\
\hline 1-reciprocity & $0.000<0.001$ \\
\hline 2-scarcity & 0.029 \\
\hline 3-authority & $0.000<0.001$ \\
\hline 4-social proof & .012 \\
\hline 5-liking & 0.447 \\
\hline 6-commitment & 0.570 \\
\hline 0.600 & \\
0.500 & \\
0.400 & \\
0.200 & \\
0.100 & \\
0.000 & \\
\hline
\end{tabular}

Fig. 2 the effective of six strategies

The results fig. 3 indicated that explanations based upon the strategies reciprocity and authority have proven to be more effective compared to the other strategies .

\section{CONCLUSIONS}

In this research we use a modified recommendation system that combines using a traditional recommendation system and using a persuasion explanation recommendation system, we conclude that a list of recommendation items will suggest to the user and he will persuade with it . We use persuasion principles, we conclude the principles of Authority and reciprocity revealed as the most powerful principles and more effective compared to the other strategies. We use persuasion explanations in a recommendation system, we conclude that they increased user's intention to use a recommended items even if this item is not on their interests. Furthermore, the above results provide only for the book recommendation domain, in which the recommended items (books) present certain characteristics, we conclude that our system are applicable to other domains (e.g. other product categories).

In this research, we focused on books that users were actually not interested in, We conclude that we measure differences in the users' intention to read. However, users expect items similar to their interests to be proposed by a recommendation system, we conclude that the potential effect of such expectation must be controlled and measured.

\subsection{FUTURE WORK}

In our future research, first we plan to increase the sample size because it is rather small to derive conclusive results. Further extension of the experiment to a larger and more diverse group of user will provide additional validity support to the findings.

Furthermore, we plan to apply the same experimental process on books where users have expressed high levels of intention to use and compare the findings with the ones of the present study (on items with low intention to use) Because enhancing the influence of recommendations utilizing the influence principles should not violate the basic purpose of recommender systems to support users in their decision making process and also act as marketing promotional vehicles. Finally, I hope that such systems that depend on 
the engineering of persuasion to activate in the Egyptian society despite the difficulties to achieve the benefits of which is the luxury of people.

\section{REFERENCES}

[1] Lipi S., Hetal G.,Prem B., “ Survey on Recommendation System”, International Journal of Computer Applications (0975 - 8887) Volume 137 - No.7, March (2016).

[2] Athulya R., Remya R. ,'International Journal of Computer Applications” (0975 - 8887) Volume 133 No.15, January 2016 A Case Study on Various Recommendation .

[3] Fogg B.," Persuasive computers: Perspectives and research directions", Proceedings of the SIGCHI conference on Human factors in computing systems CHI '98 (1998).

[4] Tintarev N. , Masthoff J., "A survey of explanations in recommender systems," in IEEE 23rd International Conference on Data Engineering Workshop, p.p 801-810, 2007.

[5] Tintarev, N. and Masthoff, J. "Effective explanations of recommendations user-centered design" Proceedings of the 2007 ACM conference on recommender systems RecSys '07, pp 153-156, 2007.

[6] Symeonidis P., Nanopoulos A., and Manolopoulos Y., "MovieExplain: A Recommender System with Explanations," RecSys '09 Proceedings of the third ACM conference on Recommender systems pp. 317320, 2009.

[7] Gedikli F., Ge M., and Jannach D., "Understanding recommendations by reading the clouds," ECWeb, Lecture Notes in Business Information Processing, p.p 196-208, 2011.

[8] Sofia G., George L.," The persuasive role of Explanations in Recommender Systems:, Second International Workshop on Behavior Change Support Systems (BCSS 2014) 59 .

[9] Tintarev N., Masthoff J.," Evaluating the effectiveness of explanation"s. User Model User-Adap Inter. pp.399-439 (2012).

[10] Herlocker J., Konstan J., Riedl J.," Explaining collaborative filtering recommendations”, CSCW 2000: 241-250 (2000).

[11] Halko S., Kientz J.," Personality and persuasive technology" An exploratory study on healthpromoting mobile applications,Persuasive technology, pp. 150-161 (2010)

[12] http://jmcauley.ucsd.edu/data/amazon/.

[13] Bollen D., Knijnenburg B.p, Willemsen, M. C., Graus, M.:Understanding choice overload in recommender systems. Proceedings of the fourth ACM conference on Recommender systems, 63-70 (2010).

[14] Cremonesi P., Garzotto F., Turrin R., " Investigating the Persuasion Potential of Recommender Systems from a Quality Perspective” An Empirical Study. ACM Trans. Interact. Intell. Syst., 2(2), 11:11:41(2012).

[15] Kaptein M., Ruyter B., Markopoulos P.," Adaptive persuasive systems" a study of tailored persuasive text messages to reduce snacking. ACM Transactions on Interactive Intelligent Systems (TiiS), 2(2)

(2012).

[16] The Apache Foundation. (2014, April 19). GenericUserBasedRecommender.java [Online].

Available:https://github.com/apache/mahout/blob/trunk/mrlegacy/src/main/java/org/apache/mahout/cf/tast e/impl/recommender/GenericUserBasedRecommender.java.

[17] Halko S., Kientz J., "Personality and persuasive technology: An exploratory study on healthpromoting mobile applications", Persuasive technology, pp. 150-161 (2010). 